\title{
Unraveling the genetic architecture of environmental variance of somatic cell score using high-density single nucleotide polymorphism and cow data from experimental farms
}

\author{
H. A. Mulder, ${ }^{*} \dagger^{1}$ R. E. Crump, $\dagger$ M. P. L. Calus, $\dagger$ and R. F. Veerkamp $\dagger$ \\ *Animal Breeding and Genomics Centre, Wageningen University, PO Box 338, 6700 AH Wageningen, the Netherlands \\ †Animal Breeding and Genomics Centre, Wageningen UR Livestock Research, PO Box 65, 8200 AB Lelystad, the Netherlands
}

\begin{abstract}
In recent years, it has been shown that not only is the phenotype under genetic control, but also the environmental variance. Very little, however, is known about the genetic architecture of environmental variance. The main objective of this study was to unravel the genetic architecture of the mean and environmental variance of somatic cell score (SCS) by identifying genome-wide associations for mean and environmental variance of SCS in dairy cows and by quantifying the accuracy of genome-wide breeding values. Somatic cell score was used because previous research has shown that the environmental variance of SCS is partly under genetic control and reduction of the variance of SCS by selection is desirable. In this study, we used 37,590 single nucleotide polymorphism (SNP) genotypes and 46,353 test-day records of 1,642 cows at experimental research farms in 4 countries in Europe. We used a genomic relationship matrix in a double hierarchical generalized linear model to estimate genome-wide breeding values and genetic parameters. The estimated mean and environmental variance per cow was used in a Bayesian multi-locus model to identify SNP associated with either the mean or the environmental variance of SCS. Based on the obtained accuracy of genome-wide breeding values, 985 and 541 independent chromosome segments affecting the mean and environmental variance of SCS, respectively, were identified. Using a genomic relationship matrix increased the accuracy of breeding values relative to using a pedigree relationship matrix. In total, $43 \mathrm{SNP}$ were significantly associated with either the mean (22) or the environmental variance of SCS (21). The SNP with the highest Bayes factor was on chromosome 9 (Hapmap31053-BTA-111664) explaining approximately $3 \%$ of the genetic variance of the environmental variance of SCS. Other significant SNP explained less than $1 \%$ of the genetic variance. It
\end{abstract}

Received March 15, 2013.

Accepted July 18, 2013.

${ }^{1}$ Corresponding author: Han.mulder@wur.nl can be concluded that fewer genomic regions affect the environmental variance of SCS than the mean of SCS, but genes with large effects seem to be absent for both traits.

Key words: genome-wide association, environmental variance, genomic selection, somatic cell score

\section{INTRODUCTION}

Mastitis is one of the most costly diseases in dairy cattle husbandry. Recording of mastitis is limited and the heritability of mastitis resistance is low. Therefore, direct genetic selection for mastitis resistance is difficult. Mastitis leads, however, to increased SCC, which is easy to measure during routine milk recording and has a higher heritability than mastitis. Therefore, SCS (log-transformed SCC) is often used as an index trait for mastitis, and in some national genetic evaluations, SCC peaks are used as predictor traits for mastitis (de Haas et al., 2008). Moreover, elevated SCC has a negative economic value, because milk factories give price penalties to farmers if bulk tank SCC is above a certain threshold (Veerkamp et al., 1998). Therefore, SCC is also a breeding goal trait in itself. Occurrence of mastitis leads to elevated SCC or peaks and, as such, also increases the lactation variance of SCS per cow (Green et al., 2004; de Haas et al., 2008; Urioste et al., 2012). Therefore, selection for reduced standard deviation or variance of SCS are alternatives that could be used to improve mastitis resistance. Urioste et al. (2012) showed that the heritability of standard deviation of SCS was 0.14 . Another way to model the variability in SCS is to model genetic differences in environmental variance between cows using a double hierarchical generalized linear model (DHGLM), as shown by Rönnegård et al. (2013). Rönnegård et al. (2013) showed that the environmental variance of SCS is heritable. The amount of genetic variance in the size of the environmental variance is, however, small. In addition, Rönnegård et al. (2013) showed that the variance of SCS has a negative economic value in its own right because of price penalties for too-high SCC by milk factories. 
Table 1. Summary statistics of SCC $(\times 1,000$ cells $/ \mathrm{mL})$ and SCS $\left[\mathrm{SCS}=\log _{2}(\mathrm{SCC})\right]$

\begin{tabular}{lccccc}
\hline Trait & $\begin{array}{c}\text { Number } \\
\text { of records }\end{array}$ & Mean & SD & Minimum & Maximum \\
\hline SCC & 46,353 & 133 & 411 & 1 & 14,678 \\
SCS & 46,353 & 5.73 & 1.65 & 0.00 & 13.84 \\
\hline
\end{tabular}

Several studies have performed searches for QTL for mastitis or SCS (Rupp and Boichard, 2003; Khatkar et al., 2004). Wijga et al. (2012) found 2 SNP associated with lactation standard deviation of SCS (i.e., on chromosome 6 and 18). The one on chromosome 18 was also significant for the lactation average of SCS. So far, no studies have estimated genome-wide associations for measures of environmental variance for SCS or for any other traits in dairy cattle (e.g., obtained from double hierarchical generalized linear models; Rönnegård et al., 2010). Individual differences in environmental variance may be considered as differences in micro-environmental sensitivity, which might be partly under genetic control. In the case of SCS, higher variance in SCS can be caused by a higher incidence of mastitis (i.e., due to the bimodal distribution of SCS for healthy and mastitic cows). Studies that have searched for QTL of environmental variance investigated traits in Arabidopsis thaliana (Shen et al., 2012), pigs (Yang et al., 2011), and chickens (Rönnegård and Valdar, 2011; Wolc et al., 2012), but so far, such studies have not been performed in dairy cattle. In addition, accuracies of pedigree-based EBV or direct genomic values (DGV) for environmental variance of SCS are unknown.

The main objective of this study was to unravel the genetic architecture of mean and environmental variance of SCS by identifying genome-wide associations for mean and environmental variance of SCS in dairy cows and by quantifying the accuracy of DGV. In addition, genetic parameters for mean and environmental variance of SCS were estimated using both a genomic relationship matrix (GRM) and a numerator or pedigree relationship matrix (NRM). Note that the name environmental variance was used because we are interested in the genetic architecture of the true environmental variance and the residual variance of the models used in our study provide an estimate of that true environmental variance.

\section{MATERIALS AND METHODS}

\section{Data}

The data used in the present study originated from Teagasc Moorepark Dairy Production Research Center (Oak Park, Carlow, Ireland), Scottish Agricultural College (Edinburgh, UK), Wageningen UR Livestock
Research (Lelystad, the Netherlands), and Swedish University of Agricultural Science (Uppsala, Sweden). On 1,642 first-lactation Holstein heifers, SCC and SCS $\left[\mathrm{SCS}=\log _{2}(\mathrm{SCC})\right]$ data of 46,353 test-day records were available, as well as genotype data. These cows had between 1 and 45 test days, with a median of 30 test days. For estimation of genetic parameters, we used all of these cows. For estimation of genome-wide associations, we used records of cows with more than 5 test days, which resulted in 1,563 cows, and the pedigree contained 9,368 animals. Summary statistics of the phenotypic data used are in Table 1.

All used animals with phenotypic information were genotyped with the Illumina BovineSNP50 BeadChip (Illumina Inc., San Diego, CA) containing 54,001 SNP. Single nucleotide polymorphisms that fulfilled the following criteria were included in the analysis: (1) gene calling (GC) score $>0.20$ and genetic testing (GT) score $>0.55$, (2) call rate $>95 \%$, (3) minor allele frequency $>0.01$ in each country, and (4) no extreme deviation from Hardy-Weinberg equilibrium (i.e., $\chi^{2}<600 ; P=$ $1.7 \times 10^{-132}$ ). After the quality control, 37,590 SNP remained. Checks for Mendelian inconsistencies between pedigree and SNP data were performed for all genotyped parent-offspring pairs and among sibs (Calus et al., 2011). Missing genotypes were imputed using BEAGLE software (Browning and Browning, 2007). Chromosome number and locations of the SNP on the BovineSNP50 were obtained from the UMD3.0 bovine genome assembly from the University of Maryland (College Park). The phenotypic and genotypic data as well as the edits were basically the same as used in van Binsbergen et al. (2012), except that the number of records and the number of animals was slightly different due to not all animals having SCC records.

\section{Prediction of (Genomic) Breeding Values and Genetic Parameters}

A DHGLM was used to estimate breeding values and genetic parameters for mean and environmental variance of test-day SCS using either NRM or GRM. The DHGLM was described by Rönnegård et al. (2010) and the implementation in ASReml (Gilmour et al., 2006) was used here. A univariate model was used iteratively on both the mean and environmental variance of SCS. Rönnegård et al. (2010) used for the variance model a 
Gamma generalized linear model (GLM) with a log link function. Linearizing the response variable for environmental variance is, however, equivalent to using a Gamma GLM with a log link function and makes it possible to use a linear model for environmental variance (Felleki et al., 2012). The linearized response variable $\psi_{i j k l}$ for each observation (Felleki et al., 2012) was calculated as

$$
\psi_{i j k l}=\log \left(\hat{\sigma}_{e_{i j k l}}^{2}\right)+\frac{\frac{\hat{e}_{i j k l}^{2}}{1-h_{i j k l}}-\hat{\sigma}_{e_{i j k l}^{2}}^{2}}{\hat{\sigma}_{e_{i j k l}}^{2}},
$$

where $\hat{\sigma}_{e_{i j k l}}^{2}$ is the predicted environmental variance for each observation, $\hat{e}_{i j k l}^{2}$ is the squared estimated residual corresponding to phenotypic record $y_{i j k l}$ (46,353 records), and $h_{i j k l}$ is the leverage, the diagonal element of the hat-matrix of $\mathbf{y}$ corresponding to $y_{i j k l}$ (Hoaglin and Welsh, 1978). The model for the mean was

$$
y_{i j k l}=\mathbf{b}^{\prime} \mathbf{x}_{i j k l}+a_{i}+p e_{i}+t_{j}+m_{k}+e_{i j k l} .
$$

The environmental variance model was

$$
\psi_{i j k l}=\mathbf{b}_{v}{ }^{\prime} \mathbf{x}_{i j k l}+a_{v_{i}}+p e_{v_{i}}+m_{v_{k}}+e_{v_{i j k l}},
$$

where $\mathbf{x}_{i j k l}$ is the incidence vector for fixed effects for observation $l$ of animal $i$ (1,642 animals) in treatment $j$ (55 levels) and management group $k$ (354 levels), $\mathbf{b}\left(\mathbf{b}_{v}\right)$ is the vector with solutions for fixed effects for $y_{i j k l}$ $\left(\psi_{i j k l}\right), a_{i}\left(a_{v_{i}}\right)$ is the additive genetic effect for mean (environmental variance) for animal $i, p e_{i}\left(p e_{v_{i}}\right)$ is the permanent environmental effect for mean (environmental variance) for animal $i, t_{j}$ is the effect for treatment $j$ effect for mean, $m_{k}\left(m_{v_{k}}\right)$ is the management group effect $k$ for mean (environmental variance), and $e_{i j k l}\left(e_{v_{i j k l}}\right)$ is the residual for mean (environmental variance). Note that in the implementation used here with a linear model, it is appropriate to use a residual effect for the environmental variance model, whereas it is not in other implementations using GLM (e.g., Rönnegård et al. 2010). Because treatment effects did not have a significant effect on the environmental variance according to a likelihood ratio test, treatment effects were excluded from the variance model. The additive genetic effects are assumed to be normally distributed either with the NRM $(\mathbf{A})$ or the GRM $(\mathbf{G}): \mathbf{a} \sim \mathrm{N}\left(0, \mathbf{A} \sigma_{a}^{2}\right)$ and $\mathbf{a}_{v} \sim \mathrm{N}\left(0, \mathbf{A} \sigma_{a_{v}}^{2}\right)$ or $\mathbf{a} \sim \mathrm{N}\left(0, \mathbf{G} \sigma_{a}^{2}\right)$ and $\mathbf{a}_{v} \sim \mathrm{N}\left(0, \mathbf{G} \sigma_{a_{v}}^{2}\right)$, where $\sigma_{a}^{2}$ is the additive genetic variance for SCS and $\sigma_{a_{v}}^{2}$ is the additive genetic variance for environmental variance of SCS. The permanent environmental effects (pe and $\mathbf{p e}_{v}$ ), treatment effects $(\mathbf{t})$, and management group effects ( $\mathbf{m}$ and $\mathbf{m}_{v}$ ) were assumed to be normally distributed as follows: $\mathbf{p e} \sim \mathrm{N}\left(0, \mathbf{I} \sigma_{p e}^{2}\right), \mathbf{p e} e_{v} \sim \mathrm{N}\left(0, \mathbf{I} \sigma_{p e_{v}}^{2}\right)$, $\mathbf{t} \sim \mathrm{N}\left(0, \mathbf{I} \sigma_{t}^{2}\right), \mathbf{m} \sim \mathrm{N}\left(0, \mathbf{I} \sigma_{m}^{2}\right)$, and $\mathbf{m}_{v} \sim \mathrm{N}\left(0, \mathbf{I} \sigma_{m_{v}}^{2}\right)$. The vectors with residuals $\mathbf{e}$ and $\mathbf{e}_{v}$ are assumed to be independent and normally distributed:

$$
\left[\begin{array}{c}
\mathbf{e} \\
\mathbf{e}_{v}
\end{array}\right] \sim \mathrm{N}\left(\begin{array}{l}
0 \\
0
\end{array},\left[\begin{array}{cc}
\mathbf{W}^{-1} \sigma_{\varepsilon}^{2} & 0 \\
0 & \mathbf{W}_{v}^{-1} \sigma_{\varepsilon_{v}}^{2}
\end{array}\right]\right),
$$

where $\mathbf{W}=\operatorname{diag}(\hat{\boldsymbol{\psi}})^{-1}$ and $\mathbf{W}_{v}=\operatorname{diag}\left(\frac{1-\mathbf{h}}{2}\right)$, and $\sigma_{\varepsilon}^{2}$ and $\sigma_{\varepsilon_{v}}^{2}$ are scaling variances, which are expected to be unity, because $\mathbf{W}$ and $\mathbf{W}_{v}$ contain already the reciprocals of the residual variances per observation. Estimating $\sigma_{\varepsilon}^{2}$ and $\sigma_{\varepsilon_{v}}^{2}$ allows for departures from the expectation of unity.

The fixed effects in the model were a fixed effect for the mean lactation curve (fourth-order polynomial), and a deviation from this mean curve for 10 management groups (first-order polynomial and the exponential part of the Wilmink curve (Wilmink, 1987). Three hundred and fifty-four management groups were formed by combinations of farm, nutritional system, and milking frequency. Specific treatment effects were fitted for experimental treatments during lactation for the cows in Ireland (55 levels).

Models 1 and 2 were run consecutively in ASReml according to the following steps:

1. Run model on $\mathbf{y}$ in Equation 1 with homogeneous residual variance $\mathbf{W}=\operatorname{diag}\left(\hat{\sigma}_{e}^{2}\right)$ in ASReml until convergence,

2. Calculate $\boldsymbol{\psi}$ and $\mathbf{W}_{v}$,

3. Run model on $\psi$ in Equation 2 in ASReml until convergence,

4. Calculate $\mathbf{W}$,

5. Run model on $\mathbf{y}$ in Equation 1 in ASReml until convergence,

6. Update $\boldsymbol{\psi}$ and $\mathbf{W}_{v}$,

7. Iterate steps 3 till 6 until convergence of estimated parameters.

One iteration consisted of steps 3 to 6 . After 30 of these iterations, variance components were virtually converged at the second decimal, but showed small oscillations between 2 consecutive iterations. Therefore, estimated variance components were calculated as the average of the last 10 iterations. 
Table 2. Variance components (SE in parentheses), heritability for mean and environmental variance of SCS, and genetic coefficient of variation (GCV) for environmental variance of SCS using a pedigree (NRM) or genomic $(\mathrm{GRM})$ relationship matrix

\begin{tabular}{lccccc}
\hline & \multicolumn{2}{c}{ Mean SCS } & & \multicolumn{2}{c}{ Environmental variance of SCS } \\
\cline { 2 - 3 } \cline { 5 - 6 } Variance/parameter & NRM & GRM & & NRM & GRM \\
\hline Management $_{\text {Treatment }}{ }^{1}$ & $0.059(0.010)$ & $0.059(0.010)$ & & $0.046(0.006)$ & $0.046(0.006)$ \\
Genetic $_{\text {Permanent environment }}$ & $0.079(0.045)$ & $0.081(0.045)$ & & & \\
Heritability $^{2}$ & $0.249(0.077)$ & $0.219(0.057)$ & & $0.078(0.033)$ & $0.048(0.023)$ \\
$h_{v}^{23}$ & 0.130 & 0.125 & & $0.443(0.035)$ & $0.470(0.030)$ \\
GCV $^{4}$ & & & & 0.010 & 0.011 \\
\hline
\end{tabular}

${ }^{1}$ Treatment effects were only estimated for mean SCS, not for environmental variance of SCS.

${ }^{2}$ The heritability for mean SCS was calculated as $h_{\text {mean }}^{2}=\sigma_{a}^{2} /\left(\sigma_{a}^{2}+\sigma_{p e}^{2}+\sigma_{e}^{2}\right)$, where $\sigma_{a}^{2}$ is the additive genetic variance for SCS, $\sigma_{p e}^{2}$ is the permanent environmental variance for SCS, and $\sigma_{e}^{2}$ is environmental variance from a model with homogenous environmental variance; the heritability for environmental variance of SCS was defined as $h_{\psi}^{2}=\sigma_{a_{v}}^{2} /\left(\sigma_{a_{v}}^{2}+\sigma_{p e_{v}}^{2}+\sigma_{e_{v}}^{2}\right)$, where $\sigma_{a_{v}}^{2}$ is the additive genetic variance for environmental variance of SCS. No approximation was available for the SE.

${ }^{3}$ Heritability of environmental variance was defined as regression of $a_{v}$ on squared phenotype (Mulder et al., 2007). No approximation was available for the SE.

${ }^{4}$ The GCV was calculated as the square root of genetic variance in environmental variance (Mulder et al., 2007). No approximation was available for the SE.

The GRM was constructed according to VanRaden (2008) using the 37,590 SNP genotypes. The GRM is subject to sampling errors because only part of the genome sequence is used (Powell et al., 2010). Therefore, the GRM was adjusted following the procedure suggested by Yang et al. (2010). The procedure of adjustments is described in more detail in Veerkamp et al. (2011).

The heritability of mean SCS was calculated as $h_{\text {mean }}^{2}=\sigma_{a}^{2} /\left(\sigma_{a}^{2}+\sigma_{p e}^{2}+\sigma_{e}^{2}\right)$, with $\sigma_{e}^{2}$ being the environmental variance from a model with homogenous environmental variance. The heritability of environmental variance of SCS was calculated in 2 ways: (1) at the level of environmental variance: $h_{\psi}^{2}=\sigma_{a_{v}}^{2} /\left(\sigma_{a_{v}}^{2}+\sigma_{p e_{v}}^{2}+\sigma_{e_{v}}^{2}\right)$, where $\sigma_{e_{v}}^{2}=\sigma_{\varepsilon_{v}}^{2} / \overline{\operatorname{diag}\left(\mathbf{W}_{v}\right)}$, the average residual variance of $\boldsymbol{\psi}$ (see Table 2), and (2) at the level of squared phenotype: $h_{v}^{2}=\frac{\sigma_{a_{v, \text { add }}}^{2}}{2 \sigma_{p}^{4}+3 \sigma_{a_{v, \text { add }}}^{2}}$, where $\sigma_{a_{v, \text { add }}}^{2}$ is the estimated additive genetic variance in environmental variance in an additive model and $\sigma_{p}^{4}$ is the squared phenotypic variance [see Mulder et al. (2007) for details and derivation]. Genetic coefficients of variation for environmental variance of SCS were approximated as the square root of $\sigma_{a_{v}}^{2}$ (Mulder et al., 2007).

\section{Cross-Validation and Number of Independent Chromosome Segments}

A 10-fold cross-validation was carried out to assess the accuracy of DGV and pedigree EBV for mean and environmental variance of SCS using the DHGLM procedure as described above, either with the GRM to obtain DGV or with the NRM to obtain EBV. In each of the 10-fold cross-validations, all records for $10 \%$ of the cows were set to be missing. Animals were assigned at random to 1 of the 10-fold cross-validations. Correlations were calculated between DGV or EBV and adjusted phenotypes [adjusted phenotype for mean SCS $\left(y^{*}\right)=a+p e+e$ and adjusted phenotype for environmental variance of SCS $\left.\left(\varphi^{*}\right)=a_{v}+p e_{v}+e_{v}\right]$. Correlations were calculated within each fold and then averaged across folds. Accuracies were calculated by dividing the correlation between DGV or EBV and adjusted phenotype by the square root of the heritability $\left(h_{\text {mean }}^{2}\right.$ and $\left.h_{\psi}^{2}\right)$ to account for the fact that phenotypic variance contains genetic, permanent environmental, and environmental variance (Verbyla et al., 2010). Based on the obtained accuracies $\left(r_{\hat{g} g}\right)$ and the number of effective phenotypes used in the training set $\left(n_{p}\right)$, the number of independent chromosome segments $\left(n_{g}\right)$ was calculated equivalent to Verbyla et al. (2010) using the formula of Daetwyler et al. (2008):

$$
n_{g}=\frac{n_{p} h^{2}\left(1-r_{\hat{g} g}^{2}\right)}{r_{\hat{g} g}^{2}} .
$$

The number of effective phenotypes was calculated accounting for repeated observations on cows as $n_{p}=$ $n m /[1+(m-1) r]$, where $n$ is the number of cows, $m$ is the average number of observations per cow and $r$ is the repeatability (see the Appendix for the deriva- 
tion). The equation of Daetwyler et al. (2008) can be reformulated to calculate the accuracy as a function of heritability, repeatability, number of animals, and the average number of observations per animal as

$$
r_{\hat{g} g}=\sqrt{\frac{n m h^{2}}{n m h^{2}+n_{g}[1+(m-1) r]}} .
$$

\section{Estimation of Genome-Wide Associations}

Three traits were derived to perform genome-wide associations using univariate Bayesian stochastic search variable selection (SSVS; Calus et al., 2008; Verbyla et al., 2010). The first trait was the mean SCS per cow, calculated as the average SCS across test days per cow. In addition, we calculated the environmental variance per cow using the residuals of the last ASReml run of the DHGLM analysis with either the pedigree (VareNRM) or the genomic relationship (VareGRM) matrix. The environmental variance was calculated per cow as the mean of the squared residuals divided by 1 minus the leverage $\left(h_{i j k l}\right)$, the diagonal element of the hat-matrix of $\mathbf{y}$ corresponding to $y_{i j k l}$ (Hoaglin and Welsh, 1978): $\hat{\sigma}_{e}^{2}=\sum_{i}^{m} \frac{\hat{e}_{i j k l}^{2}}{1-h_{i j k l}} / m$, where $m$ is the number of test days of the cow. Prior to Bayesian SSVS, these 3 traits were preadjusted for fixed management group effects and random management group-year effects using ASReml. We have used preadjusted means to find genome-wide associations, which is similar to using deregressed EBV, because deregression and removing the parent average would yield similar results as using these preadjusted means because most genotyped cows would have few or no offspring. Because of strong deviations from normality, VareNRM and VareGRM were log-transformed before the preadjustment (see Figure 1 for histogram).

The univariate Bayesian SSVS model was (Calus et al., 2008; Verbyla et al., 2010) as follows:

$$
y_{i}=\mu+a_{i}+\sum_{j=1}^{n l o c} \sum_{k=1}^{2} \mathrm{SNP}_{i j k}+e_{i},
$$

where $y_{i}$ is the phenotypic record for animal $i, \mu$ is the overall mean, $a_{i}$ is the random polygenic effect of animal $i$, nloc is is the number of $\mathrm{SNP}, \mathrm{SNP}_{i j k}$ is a random effect for allele $k$ at locus $j$ of animal $i$, and $e_{i}$ is a random residual for animal $i$. The SNP effects, denoted as $\mathrm{SNP}_{i j k}$, were estimated as $q_{i j k} \times v_{k}$, following Meuwissen and Goddard (2004), where $q_{i j k}$ is the size of the effect of allele $k$ at locus $j$ and $v_{k}$ is a scaling factor in the direction vector for locus $k\left(v_{k}\right)$. More details on the prior distributions and the fully conditional distributions can be found in Meuwissen and Goddard (2004). All parameters were estimated with a Markov Chain Monte Carlo method using Gibbs sampling with residual updating. For each trait, 3 Gibbs chains of 50,000 iterations were run, discarding 10,000 iterations for burn-in. Posterior probabilities averaged across the 3 chains were converted into Bayes factors $(\mathrm{BF})$ as follows: $\mathrm{BF}=P\left(\mathrm{SNP}_{j} \mid y\right)\left[1-P\left(\mathrm{SNP}_{j}\right)\right] /\left\{\left[1-P\left(\mathrm{SNP}_{j} / y\right)\right]\right.$ $\left.P\left(\mathrm{SNP}_{j}\right)\right\}$, where $P\left(\mathrm{SNP}_{j} \mid y\right)$ is the posterior probability and $P\left(\mathrm{SNP}_{j}\right)$ is the prior probability of SNP $j$ to be associated with $y$ [see, for example, Kass and Raftery (1995)]. The posterior proportion of genetic variance explained per SNP was calculated as $2 p q \alpha^{2}$ divided by the sum of all SNP variances, where $p(q)$ is the allele frequency of one (or the other) SNP allele and $\alpha$ is the posterior mean allele substitution effect. The calculated posterior genetic variance per SNP was averaged across the 3 chains.

\section{RESULTS}

\section{Genetic Parameters for Mean and Environmental Variance of SCS}

Table 2 shows the variance components estimated with the DHGLM using either NRM or GRM. Variance components were generally very similar for both analyses. The main difference was that the additive genetic variances for both the mean and the environ-

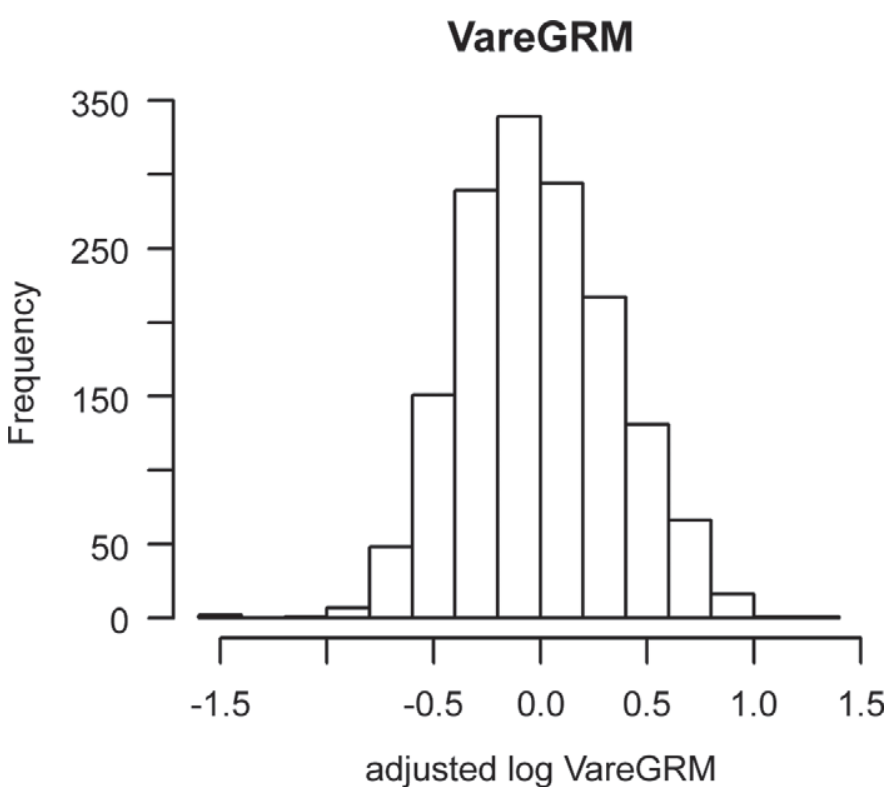

Figure 1. Histogram of log-transformed environmental variance of SCS using a genomic relationship matrix (VareGRM) and adjusted for systematic environmental effects. 
Table 3. Correlations between direct genomic value (DGV) or EBV with phenotype, accuracies of DGV and $\mathrm{EBV}$, and number of independent chromosome segments for the mean of SCS and the environmental variance of SCS (SD in parentheses)

\begin{tabular}{lcc}
\hline & & Trait \\
\cline { 2 - 3 } Parameter & Mean SCS & $\begin{array}{c}\text { Environmental } \\
\text { variance SCS }\end{array}$ \\
\hline Correlation: DGV and phenotype & $0.154(0.046)$ & $0.041(0.028)$ \\
Correlation: EBV and phenotype & $0.132(0.046)$ & $0.041(0.032)$ \\
Accuracy of DGV & $0.437(0.131)$ & $0.400(0.271)$ \\
Accuracy of EBV & $0.366(0.128)$ & $0.310(0.240)$ \\
Number of chromosome segments & $985(1,163)$ & $541(8,808)$ \\
\hline
\end{tabular}

mental variance were lower and the permanent environmental variances were higher when using a GRM in comparison to using a NRM. Differences were not significant, however. The sum of the variance components was approximately the same, indicating that the partitioning between genetic and permanent environmental variance was slightly different. The estimated per-cow environmental variances based on NRM or GRM and used in the genome-wide association study were highly correlated $(\mathrm{r}>0.999)$, as were the residuals themselves, indicating that the estimation of fixed and random effects was very similar between both models. The genetic variance in environmental variance of SCS was moderate. Standard errors were lower when using the GRM compared with using the NRM, especially for the genetic variance and the permanent environmental variance, implying improved precision of estimates, accounting for the relationship between standard error and the size of the variance component.

\section{Accuracy of DGV}

Correlations between DGV or EBV and phenotypes were respectively 0.154 and 0.132 for mean SCS and 0.041 for environmental variance of SCS (Table 3). Accuracies were 0.437 and 0.366 for, respectively, DGV and EBV of mean SCS, whereas accuracies were 0.400 and 0.310, respectively, for DGV and EBV of environmental variance of SCS. This indicates that use of a genomic relationship matrix in a DHGLM context increases accuracy of selection for both mean and environmental variance of SCS. Standard deviations on accuracies were large, especially for the environmental variance of SCS. Because of the large variability in accuracies, high variability was also observed in the derived number of independent chromosome segments. Nevertheless, both mean and environmental variance of SCS seem to be affected by many chromosome segments. It can be concluded that using a GRM increases the accuracy of selection for both mean and environmental variance of SCS compared with using an NRM.

\section{Genome-Wide Associations for Mean SCS and Environmental Variance of SCS}

The Bayes factors of all SNP for the associations with mean SCS, environmental variance based on NRM (VareNRM), and environmental variance based on GRM (VareGRM) are illustrated in Figure 2. Table 4 shows the number of SNP per class of evidence based on Bayes factor for mean SCS, VareNRM, and VareGRM. In total, 43 SNP had strong (Bayes factor = $10-30$ ), very strong (Bayes factor $=30-100$ ), or decisive (Bayes factor $>100$ ) evidence of being associated with 1 or more traits. From these 43 SNP, 11 SNP were associated with both VareNRM and VareGRM. No overlap existed in significant SNP between VareNRM or VareGRM with mean SCS. The 4 SNP with very strong or decisive evidence were on chromosome 3, 7, 9, and 25 (Table 5). The SNP on chromosome 9 was associated with both VareNRM and VareGRM. The genetic variance explained was lower than $1 \%$ for the 4 SNP in Table 4, except the SNP Hapmap31053-BTA-111664, which explained 2.1 and $3.5 \%$, respectively, of the genetic variance for VareNRM and VareGRM. Table 6 shows the 43 SNP with strong-to-decisive evidence for 1 of the traits. It can be concluded that some SNP are associated with mean or with environmental variance of SCS.

\section{DISCUSSION}

\section{Genetic Parameters and Breeding Values}

The main aim of this study was to unravel the genetic architecture of mean and environmental variance of SCS by identifying genome-wide associations and quantifying the accuracy of genome-wide breeding values for mean and environmental variance of SCS in dairy cows. In addition, genetic parameters were estimated for mean and environmental variance of SCS with an NRM and GRM. Results show clear evidence for genetic control of both the mean and the variance of SCS, as indicated by the estimated genetic variance 

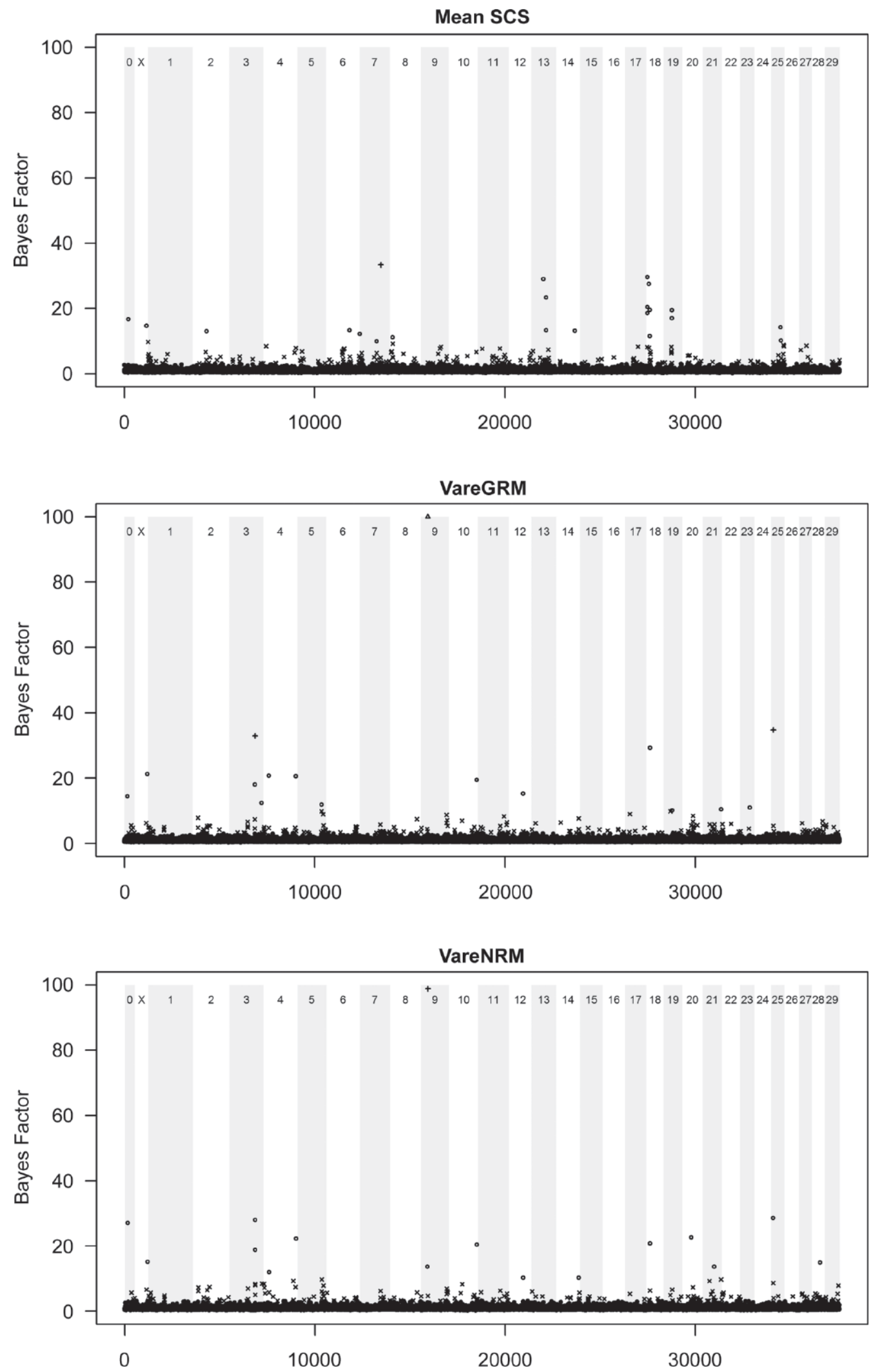

Figure 2. Manhattan plot of Bayes factors for SNP for mean SCS and environmental variance based on a genomic relationship matrix (VareGRM) or pedigree relationship matrix (VareNRM). For SNP Hapmap31053-BTA-111664, the Bayes factor was 134.7 with VareGRM, which was set to 100 in the graph of VareGRM. Chromosome numbers are indicated at the top. 
Table 4. Number of SNP loci per class of evidence based on Bayes factor for mean SCS, the environmental variance based on a genomic relationship matrix (VareGRM), and the environmental variance based on a pedigree relationship matrix (VareNRM)

\begin{tabular}{lcrrr}
\hline & & \multicolumn{3}{c}{ Trait } \\
\cline { 3 - 5 } Evidence $^{1}$ & $\begin{array}{c}\text { Bayes } \\
\text { factor }^{1}\end{array}$ & Mean SCS & VareGRM & VareNRM \\
\hline Weak & $0-3.2$ & 37,373 & 37,402 & 37,406 \\
Substantial & $3.2-10$ & 195 & 172 & 168 \\
Strong & $10-30$ & 21 & 13 & 15 \\
Very strong & $30-100$ & 1 & 1 & 1 \\
Decisive & $>100$ & 0 & 1 & 0 \\
\hline
\end{tabular}

${ }^{1}$ Evidence and ranges for Bayes factors per class are as defined by Jeffreys (1961).

in mean and environmental variance. Results using an NRM or GRM were very similar. The heritability for mean SCS was similar to other studies (Heringstad et al., 2000; Rupp and Boichard, 2003). The estimate of genetic variance in environmental variance of SCS using a GRM was similar to the estimate that Rönnegård et al. (2013) reported in Swedish Holstein using an NRM. The estimate when using an NRM was, however, higher. Genetic coefficients of variation and heritabilities for environmental variance at the level of the squared phenotype, as defined in Mulder et al. (2007), were in the low end of the range summarized by Hill and Mulder (2010). Results indicated strong permanent environmental effects on environmental variance, but lower than in Rönnegård et al. (2013). This implies that some cows have much larger fluctuations in SCS than others, which may relate to a higher incidence of (sub)clinical mastitis.

In this study, we estimated breeding values both with an NRM and a GRM in a DHGLM framework. This is the first time that genome-wide breeding values for environmental variance have been predicted with a DHGLM model and for the first time, the accuracies of pedigree and genome-wide breeding values have been assessed with cross-validation. The model showed that the accuracy of genome-wide breeding values for mean and environmental variance of SCS was higher than with pedigree-based breeding values. The predictive ability of pedigree and genome-wide breeding values in addition to the nonzero heritabilities showed that environmental variance was under genetic control.

\section{Genetic Architecture}

The numbers of independent chromosome segments derived from the accuracies of DGV were very similar for the mean and the environmental variance of SCS. The modification of the Daetwyler et al. (2008) formula allowed for the use of repeated observations. The number of independent chromosome segments was in the same range reported in the literature for other quantitative traits (Verbyla et al., 2010; Pimentel et al., 2011). The benefit of repeated observations was much larger for the environmental variance than for the mean of SCS, which is illustrated by predicting accuracies considering different numbers of animals and 1 or 30 observations per animal (Figure 3). Using the estimated number of independent chromosome segments in Table 3 and Equation 3, accuracies of 0.75 can be achieved either with 10,000 genotyped cows, each with 30 observations, or with approximately 65,000 genotyped cows with single observations. This shows that use of data from cows at experimental farms with frequent recording may reduce costs of genotyping. These calculations and the large standard deviations on accuracies and number of independent chromosome segments in the cross-validation show that the current data set is small. In the near future, it should be possible to get refer-

Table 5. Chromosome number, name of SNP, position, Bayes factor, and the proportion of genetic variance explained for the 4 SNP that have very strong or decisive evidence to be associated with mean SCS, the environmental variance based on a pedigree relationship matrix (VareNRM), and the environmental variance based on a genomic relationship matrix (VareGRM)

\begin{tabular}{lrlrrc}
\hline Trait & BTA & SNP & Location (bp) & $\begin{array}{r}\text { Bayes } \\
\text { factor }\end{array}$ & $\begin{array}{c}\text { Proportion of } \\
\text { genetic variance }\end{array}$ \\
\hline Mean SCS & 7 & ARS-BFGL-NGS-105066 & $77,513,074$ & 33.4 & 0.008 \\
VareNRM & 9 & Hapmap31053-BTA-111664 & $27,017,136$ & 98.8 & 0.021 \\
VareGRM & 9 & Hapmap31053-BTA-111664 & $27,017,136$ & 134.7 & 0.035 \\
VareGRM & 3 & BTA-28541-no-rs & $96,955,478$ & 32.9 & 0.006 \\
VareGRM & 25 & Hapmap32191-BTC-019394 & $6,103,549$ & 34.7 & 0.005 \\
\hline
\end{tabular}


Table 6. Chromosome number, name of SNP, position, and Bayes factor for the SNP that have strong, very strong, or decisive evidence of being associated with mean SCS, the environmental variance based on a pedigree relationship matrix (VareNRM), and the environmental variance based on a genomic relationship matrix (VareGRM)

\begin{tabular}{|c|c|c|c|c|}
\hline Trait & $\mathrm{BTA}^{1}$ & SNP & Location $^{1}$ (bp) & $\begin{array}{l}\text { Bayes } \\
\text { factor }\end{array}$ \\
\hline Mean SCS & 0 & ARS-BFGL-BAC-13205 & 0 & 16.8 \\
\hline Mean SCS & $\mathrm{X}$ & ARS-BFGL-NGS-83961 & $140,370,764$ & 14.7 \\
\hline Mean SCS & 2 & BTB-00098912 & $50,506,143$ & 13.1 \\
\hline Mean SCS & 6 & ARS-BFGL-NGS-28041 & $89,251,522$ & 13.4 \\
\hline Mean SCS & 7 & ARS-BFGL-NGS-19919 & 428,335 & 12.3 \\
\hline Mean SCS & 7 & BTB-00314819 & $63,279,514$ & 10.0 \\
\hline Mean SCS & 7 & ARS-BFGL-NGS-105066 & $77,513,074$ & 33.4 \\
\hline Mean SCS & 8 & ARS-BFGL-NGS-17642 & $11,307,970$ & 11.2 \\
\hline Mean SCS & 13 & ARS-BFGL-NGS-3126 & $45,112,117$ & 29.0 \\
\hline Mean SCS & 13 & Hapmap54192-rs29025257 & $53,719,704$ & 13.3 \\
\hline Mean SCS & 13 & BFGL-NGS-110706 & $53,898,040$ & 23.4 \\
\hline Mean SCS & 14 & ARS-BFGL-NGS-70724 & $63,894,637$ & 13.2 \\
\hline Mean SCS & 18 & ARS-BFGL-NGS-76223 & $5,101,055$ & 18.6 \\
\hline Mean SCS & 18 & ARS-BFGL-NGS-79168 & $5,124,435$ & 29.6 \\
\hline Mean SCS & 18 & BTA-43624-no-rs & $5,146,517$ & 20.5 \\
\hline Mean SCS & 18 & BTA-44490-no-rs & $9,515,675$ & 27.6 \\
\hline Mean SCS & 18 & BFGL-NGS-110287 & $12,168,008$ & 11.6 \\
\hline Mean SCS & 18 & BFGL-NGS-118298 & $13,029,951$ & 19.6 \\
\hline Mean SCS & 19 & ARS-BFGL-NGS-11488 & $30,220,186$ & 19.5 \\
\hline Mean SCS & 19 & BTB-01316060 & $30,340,650$ & 17.0 \\
\hline Mean SCS & 25 & Hapmap31593-BTC-028730 & $31,319,452$ & 14.3 \\
\hline Mean SCS & 25 & BFGL-NGS-119914 & $31,892,337$ & 10.2 \\
\hline VareGRM & 0 & ARS-BFGL-NGS-22958 & 0 & 14.4 \\
\hline VareGRM & $\mathrm{X}$ & ARS-BFGL-NGS-103266 & $145,497,465$ & 21.2 \\
\hline VareGRM & 3 & Hapmap52129-rs29016142 & $96,882,682$ & 18.0 \\
\hline VareGRM & 3 & BTA-28541-no-rs & $96,955,478$ & 32.9 \\
\hline VareGRM & 3 & ARS-BFGL-NGS-1295 & $116,331,635$ & 12.4 \\
\hline VareGRM & 4 & Hapmap26962-BTA-141949 & $20,335,110$ & 20.7 \\
\hline VareGRM & 4 & Hapmap54131-rs29019697 & $117,523,938$ & 20.6 \\
\hline VareGRM & 5 & ARS-BFGL-NGS-106674 & $106,296,860$ & 11.9 \\
\hline VareGRM & 9 & Hapmap31053-BTA-111664 & $27,017,136$ & 134.7 \\
\hline VareGRM & 10 & BTB-01566381 & $100,644,826$ & 19.4 \\
\hline VareGRM & 12 & Hapmap42162-BTA-26965 & $56,774,667$ & 15.2 \\
\hline VareGRM & 18 & ARS-BFGL-NGS-101491 & $13,839,646$ & 29.2 \\
\hline VareGRM & 19 & ARS-BFGL-NGS-36291 & $31,382,204$ & 10.1 \\
\hline VareGRM & 21 & ARS-BFGL-NGS-37484 & $68,683,196$ & 10.5 \\
\hline VareGRM & 23 & ARS-BFGL-NGS-63422 & $40,440,746$ & 10.9 \\
\hline VareGRM & 25 & Нарmap32191-ВТC-019394 & $6,103,549$ & 34.7 \\
\hline VareNRM & 0 & ARS-BFGL-NGS-22958 & 0 & 27.1 \\
\hline VareNRM & $\mathrm{X}$ & ARS-BFGL-NGS-103266 & $145,497,465$ & 15.2 \\
\hline VareNRM & 3 & Hapmap52129-rs29016142 & $96,882,682$ & 18.8 \\
\hline VareNRM & 3 & BTA-28541-no-rs & $96,955,478$ & 28.0 \\
\hline VareNRM & 4 & Нарmар26962-ВТА-141949 & $20,335,110$ & 12.0 \\
\hline VareNRM & 4 & Hapmap54131-rs29019697 & $117,523,938$ & 22.3 \\
\hline VareNRM & 9 & ARS-BFGL-NGS-31500 & $24,447,463$ & 13.7 \\
\hline VareNRM & 9 & Hapmap31053-BTA-111664 & $27,017,136$ & 98.8 \\
\hline VareNRM & 10 & BTB-01566381 & $100,644,826$ & 20.4 \\
\hline VareNRM & 12 & Hapmap42162-BTA-26965 & $56,774,667$ & 10.3 \\
\hline VareNRM & 14 & ARS-BFGL-NGS-98209 & $80,618,301$ & 10.3 \\
\hline VareNRM & 18 & ARS-BFGL-NGS-101491 & $13,839,646$ & 20.8 \\
\hline VareNRM & 20 & BTB-00777615 & $34,079,970$ & 22.7 \\
\hline VareNRM & 21 & Hapmap47533-ВTA-16340 & $43,787,368$ & 13.6 \\
\hline VareNRM & 25 & Hapmap32191-BTC-019394 & $6,103,549$ & 28.6 \\
\hline VareNRM & 28 & BTA-63984-no-rs & $30,070,377$ & 14.9 \\
\hline
\end{tabular}

${ }^{1} 0$ values indicate that this SNP was not mapped in the UMD3.0 bovine genome assembly (University of Maryland, College Park). 


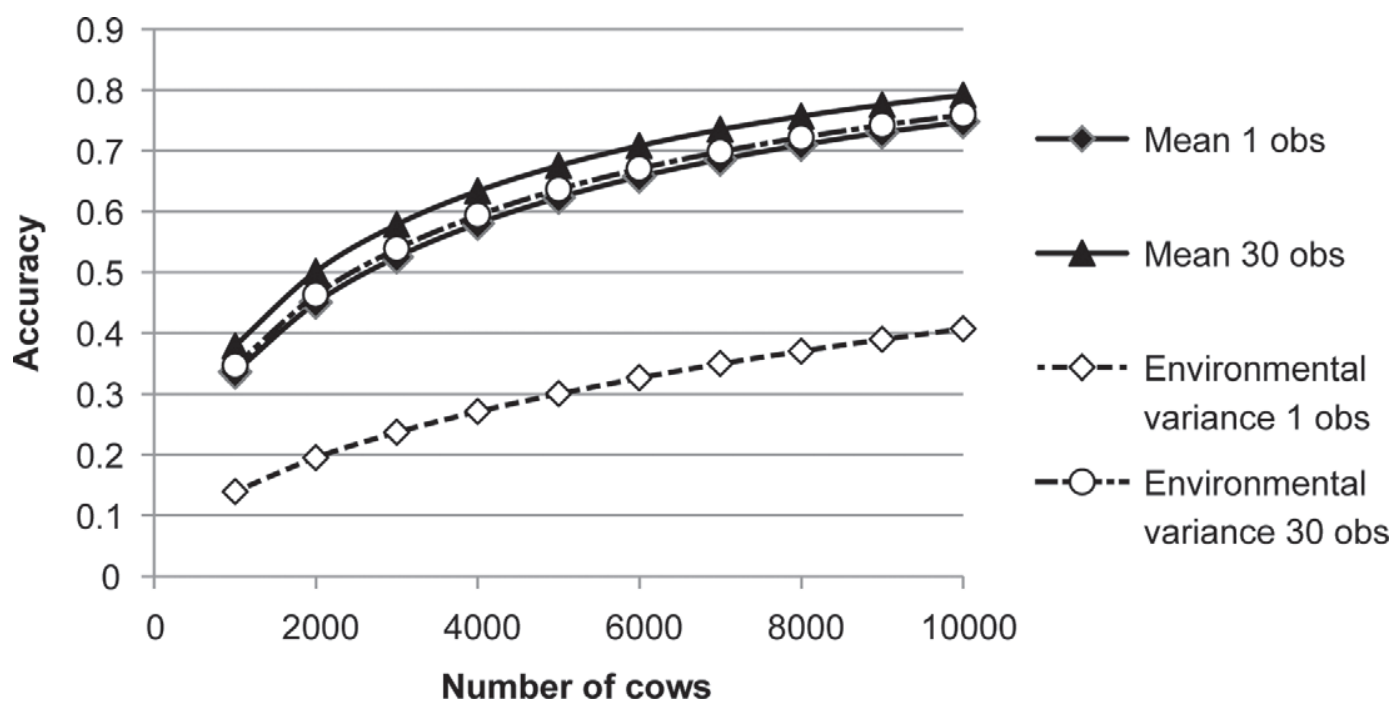

Figure 3. Predicted accuracy of direct genomic breeding value for mean SCS and environmental variance of SCS as a function of the number of cows, assuming either 1 or 30 observations (obs) per cow.

ence populations of 10,000 cows when combining data of multiple research farms across the world.

\section{Genome-Wide Associations}

For all traits, some significant SNP were found (i.e., 43 with Bayes factor above 10). The discussion is limited to the 4 significant SNP listed in Table 4 and a few that had high Bayes factors (10-30) that were also found in other studies. The SNP BTA-28541-no-rs (position 96955478) on chromosome 3 was associated with VareGRM, which was close to the marker BR4502 found in Klungland et al. (2001) to be associated with clinical mastitis and the SNP BTB-00142919 and Hapmap41332-BTA-68635 found in Cole et al. (2011) to be associated with SCS. The SNP ARS-BFGLNGS-105066 (position 77513074) on chromosome 7 was highly associated with the mean of SCS. Earlier, Tal-Stein et al. (2010) and Kühn et al. (2003) reported QTL at chromosome 7 at different locations to be associated with SCS. The SNP BTB-00314819 (position 63279514 ) with a Bayes factor of 10.0 was, however, very close to a region reported by Kühn et al. (2003) to be associated with SCS. The SNP Hapmap31053BTA-111664 (position 27739861) on chromosome 9 was highly significant for both VareGRM and VareNRM. This SNP is not very close to previously reported areas related to mastitis or SCS on chromosome 9. Lund et al. (2008) found a QTL for mastitis at $10 \mathrm{Mb}$ and Sørensen et al. (2008) found a QTL at $13.6 \mathrm{cM}$ and $15.3 \mathrm{cM}$ for pathogen-specific mastitis (marker bracket unknown). On chromosome 13, a significant SNP ARS-BFGL-NGS-3126 (position 45112117) was found located between the genes Krüppel-like factor 6 and pitrilysin metallopeptidase 1 , not too far from the region reported in Cole et al. (2011) for SCS at position 5239814. At other locations on chromosome 13, Lund et al. (2008) reported a QTL for SCS. Some significant SNP were found on chromosome 18 at approximately $51 \mathrm{Mb}$, close to the SNP ARS-BFGL-NGS-63460 (position 49966162) reported in Cole et al. (2011) to be associated with SCS, whereas other reported QTL are in proximity with the microsatellite marker TGLA 227 at around $65 \mathrm{Mb}$ found to be associated with SCS and mastitis (Kühn et al., 2003; Schulman et al., 2004). The SNP ARS-BFGL-NGS-101491 (position 13839646) was highly significant in our study for VareNRM and VareGRM, which was also found to be associated with lactation standard deviation of SCS in Wijga et al. (2012) on the same data. This region was not found in earlier studies, although several QTL were reported on BTA18 for SCS and mastitis (Khatkar et al., 2004; Schulman et al., 2004; Lund et al., 2007). The SNP Hapmap32191-BTC-019394 on chromosome 25 (position 6103549) associated with VareGRM was within the QTL region reported by Holmberg and AnderssonEklund (2004) for clinical mastitis. Also, Cole et al. (2011) reported some significant SNP at chromosome 25 for SCS, but at other locations.

Analyzing the environmental variance of SCS yielded regions that were associated with clinical mastitis or SCS in earlier studies and new regions. It is striking that even though the phenotypic correlation between VareNRM and VareGRM was $>0.99$, some different SNP were found to be significant. Although the overlap in significant SNP seems low, the correlation between 
Bayes factors was 0.87 , which is higher than the correlation between Bayes factors among individual chains within VareNRM or VareGRM. Therefore, the difference in results between VareNRM and VareGRM was more a result of between-chain variation than betweentrait variation, as both traits were almost the same. Differences occurred mainly due to the fact that SNP for one trait pass the threshold of Bayes factor $>10$, but not for the other trait. Using the GRM seemed to improve the precision of the environmental variance, which resulted in higher significance levels (e.g., for SNP Hapmap31053-BTA-111664 at chromosome 9), with Bayes factors of 135 and 99 for, respectively, VareGRM and VareNRM. In situations with fewer observations per animal, the benefit of a GRM compared with an NRM is expected to be larger, as the difference in accuracy of EBV is larger and, therefore, also the accuracy of the environmental variance. Using a GRM is expected to increase the power to detect SNP for environmental variance. A larger number of genotyped cows should also increase the power to detect SNP associated with environmental variance.

\section{CONCLUSIONS}

This study shows that the environmental variance of SCS is heritable by showing the presence of genetic variance, predictive ability of breeding values in crossvalidation, and genome-wide associations with SNP. Using a GRM increased the accuracy of breeding values relative to an NRM. Some differences in genetic parameters were observed. Based on the obtained accuracy, it was estimated that respectively 985 and 541 independent chromosome segments affected the mean and environmental variance of SCS. In total, 43 SNP were significantly associated with either the mean (22 SNP) or the environmental variance of SCS (21). The SNP with the highest Bayes factor was on chromosome 9 (Hapmap31053-BTA-111664), explaining approximately $3 \%$ of the genetic variance of the environmental variance of SCS. Other significant SNP explained less than $1 \%$ of the genetic variance. It can be concluded that fewer genomic regions affect the environmental variance of SCS than the mean of SCS, but genes with large effects seem to be absent for both traits.

\section{ACKNOWLEDGMENTS}

This project was financed by the RobustMilk project, which is financially supported by the European Commission (Brussels, Belgium) under the Seventh Research Framework Programme, Grant Agreement KBBE-211708. The content of this paper is the sole responsibility of the authors, and it does not necessarily represent the views of the Commission or its services.

\section{REFERENCES}

Browning, S. R., and B. L. Browning. 2007. Rapid and accurate haplotype phasing and missing-data inference for whole genome association studies by use of localized haplotype clustering. Am. J. Hum. Genet. 81:1084-1097.

Calus, M. P. L., T. H. E. Meuwissen, A. P. W. De Roos, and R. F. Veerkamp. 2008. Accuracy of genomic selection using different methods to define haplotypes. Genetics 178:553-561.

Calus, M. P. L., H. A. Mulder, and J. W. M. Bastiaansen. 2011. Identification of Mendelian inconsistencies between SNP and pedigree information of sibs. Genet. Sel. Evol. 43:34.

Cameron, N. D. 1997. Selection indices and prediction of genetic merit in animal breeding. CAB International, Wallingford, UK.

Cole, J. B., G. R. Wiggans, L. Ma, T. S. Sonstegard, T. J. Lawlor, B. A. Crooker, C. P. Van Tassell, J. Yang, S. Wang, L. K. Matukumalli, and Y. Da. 2011. Genome-wide association analysis of thirty one production, health, reproduction and body conformation traits in contemporary US Holstein cows. BMC Genomics 12:408.

Daetwyler, H. D., B. Villanueva, and J. A. Woolliams. 2008. Accuracy of predicting the genetic risk of disease using a genome-wide approach. PLoS ONE 3:e3395.

de Haas, Y., W. Ouweltjes, J. ten Napel, J. J. Windig, and G. de Jong. 2008. Alternative somatic cell count traits as mastitis indicators for genetic selection. J. Dairy Sci. 91:2501-2511.

Felleki, M., D. Lee, Y. Lee, A. R. Gilmour, and L. Rönnegård. 2012 Estimation of breeding values for mean and dispersion, their variance and correlation using double hierarchical generalized linear models. Genet. Res. (Camb.) 94:307-317.

Gilmour, A. R., B. J. Gogel, B. R. Cullis, and R. Thompson. 2006. ASReml User Guide Release 2.0. VSN International Ltd., Hemel Hempstead, UK.

Green, M. J., L. E. Green, L. E. Schukken, A. J. Bradley, E. J. Peeler, H. W. Barkema, Y. De Haas, V. J. Collis, and G. F. Medley. 2004. Somatic cell count distributions during lactation predict clinical mastitis. J. Dairy Sci. 87:1256-1264.

Heringstad, B., G. Klemetsdal, and J. Ruane. 2000. Selection for mastitis resistance in dairy cattle: A review with focus on the situation in the Nordic countries. Livest. Prod. Sci. 64:95-106.

Hill, W. G., and H. A. Mulder. 2010. Genetic analysis of environmental variation. Genet. Res. (Camb.) 92:381-395.

Hoaglin, D. C., and R. E. Welsh. 1978. The hat matrix in regression and ANOVA. Am. Stat. 32:17-22.

Holmberg, M., and L. Andersson-Eklund. 2004. Quantitative trait loci affecting health traits in Swedish dairy cattle. J. Dairy Sci. 87:2653-2659.

Jeffreys, H. 1961. The Theory of Probability. 3rd ed. Oxford University Press, Oxford, UK.

Kass, R. E., and A. E. Raftery. 1995. Bayes factors. J. Am. Stat. Assoc. 90:773-795.

Khatkar, M. S., P. C. Thompson, I. Tammen, and H. Raadsma. 2004 Quantitative trait loci mapping in dairy cattle: Review and metaanalysis. Genet. Sel. Evol. 36:163-190.

Klungland, H., A. Sabry, B. Heringstad, H. G. Olsen, L. Gomez-Raya, D. I. Våge, I. Olsaker, J. Ødegård, G. Klemetsdal, N. Schulman, J. Vilkki, J. Ruane, M. Aasland, K. Rønningen, and S. Lien. 2001. Quantitative trait loci affecting clinical mastitis and somatic cell count in dairy cattle. Mamm. Genome 12:837-842.

Kühn, C., J. Bennewitz, N. Reinsch, N. Xu, H. Thomsen, C. Looft, G. A. Brockmann, M. Schwerin, C. Weimann, S. Hiendleder, G. Erhardt, I. Medjugorac, M. Förster, B. Brenig, F. Reinhardt, R. Reents, I. Russ, G. Averdunk, J. Blümel, and E. Kalm. 2003. Quantitative trait loci mapping of functional traits in the German Holstein cattle population. J. Dairy Sci. 86:360-368.

Lund, M. S., B. Guldbrandtsen, A. J. Buitenhuis, B. Thomsen, and C. Bendixen. 2008. Detection of quantitative trait loci in Danish 
Holstein cattle affecting clinical mastitis, somatic cell score, udder conformation traits, and assessment of associated effects on milk yield. J. Dairy Sci. 91:4028-4036.

Lund, M. S., G. Sahana, L. Andersson-Eklund, N. Hastings, A. Fernandez, N. F. Schulman, B. Thomsen, S. M. Viitala, J. L. Williams, A. Sabry, H. Viinalass, and J. H. Vilkki. 2007. Joint analysis of quantitative trait loci for clinical mastitis and somatic cell score on five chromosomes in three Nordic dairy cattle breeds. J. Dairy Sci. 90:5282-5290.

Meuwissen, T. H. E., and M. E. Goddard. 2004. Mapping multiple QTL using linkage disequilibrium and linkage analysis information and multi-trait data. Genet. Sel. Evol. 36:261-279.

Mulder, H. A., P. Bijma, and W. G. Hill. 2007. Prediction of breeding values and selection responses with genetic heterogeneity of environmental variance. Genetics 175:1895-1910.

Pimentel, E. C. G., M. Erbe, S. König, and H. Simianer. 2011. Genome partitioning of genetic variation for milk production and composition traits in Holstein cattle. Front. Genet. 2:19.

Powell, J. E., P. M. Visscher, and M. E. Goddard. 2010. Reconciling the analysis of IBD and IBS in complex trait studies. Nat. Rev. Genet. 11:800-805.

Rönnegård, L., M. Felleki, F. Fikse, H. A. Mulder, and E. Strandberg. 2010. Genetic heterogeneity of residual variance: Estimation of variance components using double hierarchical generalized linear models. Genet. Sel. Evol. 42:8.

Rönnegård, L., M. Felleki, W. F. Fikse, H. A. Mulder, and E. Strandberg. 2013. Variance component and breeding value estimation for genetic heterogeneity of residual variance in Swedish Holstein dairy cattle. J. Dairy Sci. 96:2627-2636.

Rönnegård, L., and W. Valdar. 2011. Detecting major genetic loci controlling phenotypic variability in experimental crosses. Genetics 188:435-447.

Rupp, R., and D. Boichard. 2003. Genetics of resistance to mastitis in dairy cattle. Vet. Res. 34:671-688.

Schulman, N. F., S. M. Viitala, D. J. de Koning, J. Virta, A. MäkiTanila, and J. H. Vilkki. 2004. Quantitative trait loci for health traits in Finnish Ayrshire cattle. J. Dairy Sci. 87:443-449.

Shen, X., M. Pettersson, L. Rönnegård, and Ö. Carlborg. 2012. Inheritance beyond plain heritability: Variance-controlling genes in Arabidopsis thaliana. PLoS Genet. 8:e1002839.

Sørensen, L. P., B. Guldbrandtsen, J. R. Thomasen, and M. S. Lund. 2008. Pathogen-specific effects of quantitative trait loci affecting clinical mastitis and somatic cell count in Danish Holstein cattle. J. Dairy Sci. 91:2493-2500.

Tal-Stein, R., L. Fontanesi, M. Dolezal, E. Scotti, A. Bagnato, V. Russo, F. Canavesi, A. Friedmann, M. Soller, and E. Lipkin. 2010. A genome scan for quantitative trait loci affecting milk somatic cell score in Israeli and Italian Holstein cows by means of selective DNA pooling with single- and multiple-marker mapping. J. Dairy Sci. 93:4913-4927.

Urioste, J. I., J. Franzen, J. J. Windig, and E. Strandberg. 2012. Genetic relationships among mastitis and alternative somatic cell count traits in the first 3 lactations of Swedish Holsteins. J. Dairy Sci. 95:3428-3434.

van Binsbergen, R., R. F. Veerkamp, and M. P. L. Calus. 2012. Makeup of the genetic correlation between milk production traits using genome-wide single nucleotide polymorphism information. J. Dairy Sci. 95:2132-2143.

VanRaden, P. M. 2008. Efficient methods to compute genomic predictions. J. Dairy Sci. 91:4414-4423.

Veerkamp, R. F., H. A. Mulder, R. Thompson, and M. P. L. Calus. 2011. Genomic and pedigree-based genetic parameters for scarcely recorded traits when some animals are genotyped. J. Dairy Sci. 94:4189-4197.

Veerkamp, R. F., A. W. Stott, W. G. Hill, and S. Brotherstone. 1998. The economic value of somatic cell count payment schemes for UK dairy cattle breeding programmes. Anim. Sci. 66:293-298.

Verbyla, K. L., M. P. L. Calus, H. A. Mulder, Y. De Haas, and R. F. Veerkamp. 2010. Predicting energy balance for dairy cows using high density single nucleotide polymorphism information. J. Dairy Sci. 93:2757-2764.
Wijga, S., J. W. M. Bastiaansen, E. Wall, E. Strandberg, Y. De Haas, L. Giblin, and H. Bovenhuis. 2012. Genomic associations with somatic cell score in first-lactation Holstein cows. J. Dairy Sci. 95:899-908.

Wilmink, J. B. M. 1987. Adjustment of test-day milk, fat and protein yield for age, season and stage of lactation. Livest. Prod. Sci. $16: 335-348$

Wolc, A., J. Arango, P. Settar, J. E. Fulton, N. P. O'Sullivan, R. Preisinger, D. Habier, R. Fernando, D. J. Garrick, W. G. Hill, and J. C. M. Dekkers. 2012. Genome-wide association analysis and genetic architecture of egg weight and egg uniformity in layer chickens. Anim. Genet. 43:87-96.

Yang, J., B. Benyamin, B. P. McEvoy, S. Gordon, A. K. Henders, D. R. Nyholt, P. A. Madden, A. C. Heath, N. G. Martin, G. W. Montgomery, M. E. Goddard, and P. M. Visscher. 2010. Common SNPs explain a large proportion of the heritability for human height. Nat. Genet. 42:565-569.

Yang, Y., O. F. Christensen, and D. Sorensen. 2011. Use of genomic models to study genetic control of environmental variance. Genet. Res. (Camb.) 93:125-138.

\section{APPENDIX}

The number of effective phenotypes $n_{p}$ was calculated in such a way that the variance among the phenotypes of $n_{p}$ unrelated animals is equal to the variance of $n$ animals each with $m$ observations. Using selection index theory, the variance of phenotypes is the $\mathbf{P}$ matrix (Cameron, 1997). In the case of 2 cows, each with 2 observations and assuming a phenotypic variance of 1.0 and a correlation between the repeated observations on the same animal (the repeatability, $r$ ), $\mathbf{P}$ is blockdiagonal:

$$
\mathbf{P}=\left[\begin{array}{cccc}
1.0 & r & 0 & 0 \\
r & 1.0 & 0 & 0 \\
0 & 0 & 1.0 & r \\
0 & 0 & r & 1.0
\end{array}\right]
$$

Therefore, the variance of the average of $n$ animals each with $m$ observations is

$$
\operatorname{var}(\bar{P})=\frac{n m+n m(m-1) r}{(n m)^{2}}=\frac{1+(m-1) r}{n m} .
$$

The variance of $n_{p}$ unrelated animals each with a single observation is

$$
\operatorname{var}(\bar{P})=1 / n_{p} .
$$

When equating A2 and A3, we can derive an expression for $n_{p}$ as a function of $n, m$, and $r$ :

$$
n_{p}=n m /[1+(m-1) r] .
$$

